\title{
Raising the Dimensions and Variables for Searching as a Learning Process: A Systematic Mapping of the Literature
}

\author{
Marcelo Machado, Paulo J. A. Gimenez, Sean W. M. Siqueira \\ ${ }^{1}$ Programa de Pós-Graduação em Informática (PPGI) \\ Universidade Federal do Estado do Rio de Janeiro (UNIRIO) \\ Av. Pasteur, 456, Urca - Rio de Janeiro - RJ - Brasil \\ \{marcelo.machado, paulo.gimenez\}@edu.unirio.br, sean@uniriotec.br
}

\begin{abstract}
Search engines are great allies in our daily educational tasks. However, usually, these tools are designed for factual learning and are less effective when dealing with more complex learning tasks. For this reason, in recent years, Searching as Learning (SAL) research area has been developing from studies that target the challenges involving learning during the search process. It is known that the effectiveness of educational technologies in providing appropriate instructions depends on the input information. Gathering information on what should be taken into account in a searching as a learning process can support the development of specialized search engines to support higher levels of learning. Thus, we performed a systematic mapping of the literature to gather this information, raising the dimensions and their associated variables.
\end{abstract}

\section{Introduction}

Using the Internet to access information is a common activity in our daily lives, and search engines are strong allies in this process. In formal education, teachers and students look for learning materials (e.g., learning objects and open educational resources) to use/reuse in classes, to perform tasks, or to answer questions. In an informal education setting, searches are carried out to clarify the most varied doubts, supporting users' knowledge construction in different domains. Then, learning tasks differ according to the search intent and present distinct complexities that promote divergences in search behavior and outcome. For instance, to learn the meaning of a word, a simple query containing the word itself should be enough to recover what is necessary for the understanding. However, acquiring sufficient knowledge about machine learning models to decide which one to use, regarding diverse characteristics of a dataset, requires an iterative process of searching and analysis of the results - especially if the user has no prior knowledge on the subject.

Traditional search systems, such as Google, can and should be used for educational purposes. However, those systems aim at a quick and efficient selection of documents from the search engine result page, and its factual information search purpose is not as successful at facilitating higher levels of knowledge (Taibi et al., 2017). Rieh et al. (2016) pointed out two contradictory behaviors of users who are searching with learning purposes. On the one hand, users struggle to find the right keywords, formulate search queries and understand search. When search results do not satisfy their information needs, they tend to accept those initial results uncritically, rather than re-evaluating the results or revising their search statements. On the other hand, some studies showed that users 
overestimate their search skills and believe that accessing, searching, using and evaluating sources of information are easy tasks. As a result, these users tend to skim, evaluate and select online content quickly without investing much effort. In either case, users' behavior and search engines (as is) reveal issues to be treated to support learning.

Searching as Learning (SAL) exploits the synergy between searching and learning, focusing on the impact, influence, and consequences of using search engines as learning technologies (Machado et al., 2019). Studies of this area argue that although search engines are widely used for learning-oriented information needs, they are optimized primarily for generic relevance, not for effective learning, nor do they consider learning as an incremental process of knowledge acquisition (Gwizdka et al., 2016, Syed and CollinsThompson, 2017). In the last years, some studies have been conducted to gather an understanding of learning during search tasks to build search engines that target learning outcomes (Rieh et al., 2016). Although these studies are primarily from the Information Retrieval (IR) research area, related areas, such as Adaptive Learning and Learning Analytics, argue about the importance of the variables used as input to support learning in a Web-based system (Machado et al., 2020, Moreno-Marcos et al., 2020). Thus, the objective of this study is to identify and analyze the variables that are observed and used in SAL literature. No other study in this area organizes this kind of knowledge yet. We believe that, as well as in other research fields, this understanding may provide a basis for implementations and discussions on the correlation of these variables and learning goals.

Following a systematic literature mapping protocol, we identified 31 papers, from 2009 to 2019, that provide information about the variables involved in the searching as a learning process. Our main contributions are (i) the use of a systematic method to provide an overview of the state of the art in SAL approaches covering ten years of published papers and (ii) an analysis of the main input variables involved in SAL proposals.

The remaining of this paper is organized as follows: Section 2 reviews related works; Section 3 presents the systematic mapping methodology; Section 4 presents and discusses the variables revealed by the literature analysis. Finally, Section 5 concludes the paper and presents some perspectives of future work.

\section{Related Works}

In recent years, Searching as Learning has gained notoriety, therefore, some studies have already raised research questions about the area. In this section, we briefly present those studies that are somehow related to this research.

Rieh et al. (2016) critically review the literature on the association between searching and learning and contribute to the formulation of SAL as a research agenda. They present a perspective on SAL that focuses on the learning that occurs during the search process, as well as search outputs and learning outcomes. The authors introduce the concept of "comprehensive search" to describe iterative, reflective and integrative search sessions that facilitate critical and creative learning beyond receptive learning.

Vakkari (2016) first presents some reflections about the meaning of "learning" and "searching". Learning was conceptualized as changes in one's knowledge structures. Then it was described how learning occurs in the search process. The study related each of the defined stages of the search process to types of changes in knowledge structure 
(learning). Finally, to fulfill the objective of surveying studies, Vakkari (2016) presented different types of studies on relations between searching and learning by observing dependent and independent variables in the studies.

More recently, Hoppe et al. (2018) take a critical look at existing works in SAL and related research disciplines. They presented some issues mainly related to IR characteristics and empathized that exploring multimodal features of learning resources should be one focus of future SAL projects.

Finally, Machado et al. (2019) present a meta-analysis of the evolution of the SAL agenda based on the publications. They identify the main authors, the timeline of publications and the main research topics. This study confirms the growing interest in SAL and presents some topics commonly referenced in the publications: interaction between user and system, exploratory search, learning theories, user profile, IR, and representation of the knowledge domain.

These works were essential to support SAL as a research agenda. However, few works have proposed to discuss systematically the new proposals created on this foundation. The SAL literature served to us as a strong indicator that it is worth investigating the variables involved in the searching as a learning process. It may be a foundation for developing adequate educational technology to support learning in higher levels.

\section{Methodology}

This study followed a systematic literature review protocol in order to select and evaluate relevant studies. This review was organized based on the main activities proposed by Kitchenham and Charters (2007): planning, conducting and reporting.

In the planning activity, we defined the objectives and defined the protocol. The main objective of this research is to understand the main variables observed in dealing with a search system with a focus on learning. In order to be more specific on the aspects of searching as a learning process, our focus is exclusively on studies in the SAL research area. To meet this objective, we used the following search string ${ }^{1}$ to search papers:

\section{"Search as Learning" OR "Searching as Learning" OR "Search as a Learning" OR "Searching as a Learning"}

We chose to maintain a greater coverage in the search string (i.e. there is no term about the variables). As inclusion criteria, we selected only studies that present proposals about searching as a learning process. Theoretical studies were not included in the analyzes as our focus is on the variables involved in the processes. We also defined some exclusion criteria:

- The study is not about Searching as Learning OR

- The paper was not written in English OR

- The paper is grey literature OR

- The paper is not a full study (e.g., poster, position papers, short paper) OR

\footnotetext{
${ }^{1}$ A set of potential primary studies was defined to validate the search string accuracy and whether it was returning relevant results. "Search(ing) as a learning" was added because some papers used the terms "search(ing) as a learning activity" or even "search(ing) as a learning process".
} 
- The paper is a small extension of a different paper from the same authors (we prioritize journals over conferences papers).

After defining the search string, we proceed to the conducting activity by considering the following steps:

1. Execution of the search string in the selected digital libraries and removal of duplicates, after merging the returned results.

2. Analysis of the papers by reading (i) title and abstract, (ii) introduction and conclusion, and (iii) full text and data extraction.

3. Application of backward and forward snowballing.

Fig. 1 depicts the result obtained based on these steps. Exclusion criteria were assessed in step 2, in which the sub-steps (i) and (ii) were each applied by the first and second authors, while sub-step (iii) was further subdivided among all the authors who extracted data from the read papers. The last author analyzed divergences, doubting publications and excluded ones during sub-step (iii) in order to avoid research biases. In step 3, the backward and forward snowballing technique was applied to find some papers that were not covered by the search string. Thus, five papers were added to our dataset, totaling 31 publications.

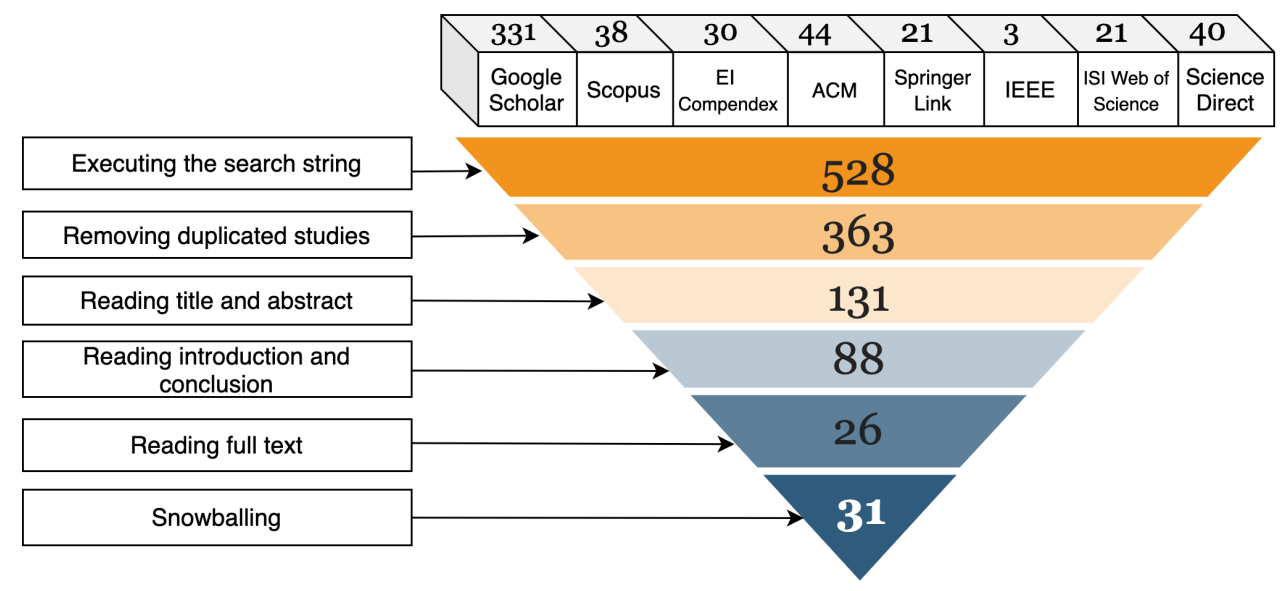

Figure 1. Results of the systematic conduction process.

\section{Gathering the input variables around Searching as Learning}

After the complete reading of the selected papers, we identified eight groups of variables. Below we describe these groups and summarize them in a proposed classification.

User Prior Knowledge $(P K)$ was largely used to represent the current learning level of the user when accessing a formal educational environment. For instance, Al-Tawil et al. (2019) assessed the user's prior knowledge about a learning concept in order to offer a utility tool aiming to increase user's domain knowledge while exploring a data graph. Besides, prior knowledge sometimes is represented by labels, such as Novice User and Expert User, on the premise that these groups have divergent seeking behavior ( $\mathrm{Lu}$ and Hsiao, 2017). Finally, another way to represent a user's prior knowledge is from cognitive traits (Taibi et al., 2017). At this point, the user is assessed from specific pretests aiming to categorize them into some taxonomy structured in levels of complexity of concepts. 
IX Congresso Brasileiro de Informática na Educação (CBIE 2020)

Anais do XXXI Simpósio Brasileiro de Informática na Educação (SBIE 2020)

Demographic Information (DI) represents basic information of a user, such as gender, spoken language, age, geographic occupation, and ethnicity. For example, Taibi et al. (2017) set up user-specific language and local settings in order to get web pages from a specific country and written in a specific language. Also, Yu et al. (2018) consider age as a factor for building more complex queries. For instance, Piaget distinguishes stages of cognitive development based on children's age (Inhelder and Piaget, 1958). Besides that, Azpiazu et al. (2017) only consider K-9 student for their solution, claiming that while the use of search engines for learning tasks enhancement is usual, they are not designed for children; thus some issues arise when it is used by this audience (Gossen et al., 2013).

Exploratory Search Activities (ESA) are variables related to the path taken by users when exploring the search space. Exploratory search requires the user's cognitive processing and interpretation through scanning/viewing, comparing, and making qualitative judgments (Marchionini, 2006). To formalize the states covered, some Information Seeking models were used, such as Marchionini's Exploratory Search Model (Marchionini, 2006), Information Foraging (Pirolli and Card, 1999), Berrypicking (Bates et al., 1989), and Exploratory Search Knowledge Intensive Process taxonomy (Tibau et al., 2019b). For instance, Tibau et al. (2018) investigate exploratory search by applying a model capable of assisting the visualization of search patterns and identifying best practices associated with users' decision-making processes. Some variables are terms selection, query formulation, query reformulation and query expansion.

Activities Variables $(A V)$ are variables based on user's navigation actions while analyzing the resources recovered by their searches. This information is usually accessed from consolidated quantitative log data. Examples of these variables are mouse usage (clicks, mouse over, scroll position, scroll distance) (Ibieta et al., 2019, Yu et al., 2018); eye-tracking data (e.g., eye-tracking fixation, reading duration) (Bhattacharya and Gwizdka, 2019, Syed and Collins-Thompson, 2018); the number of queries, query diversity, query complexity (Rieh et al., 2012); browsing (pages visited, pages saved, dwell time) (Ibieta et al., 2019); click on a search result, clicks per query (Yu et al., 2018); and session (duration of a search session, duration per query) (Yu et al., 2018). Most of the studies that observed this type of variable were quantitative. The main motivation is to find a correlation between these variables and learning.

Search Engine Feedback ( $S E F$ ) group includes variables such as query suggestions (e.g., "Did you mean" and query building while typing), word synonyms, grammar and spell checking; and other IR strategies like query expansion (Lucchese et al., 2018) and collaborative support (Soulier and Tamine, 2017). That is, it includes variables related to the behavior of the search tool towards user actions. As an example, Azpiazu et al. (2017) proposed a system - namely YUM, that presents a strategy that generates diverse kid-oriented query suggestions.

Knowledge Domain Representation (KDR) group includes variables related to the data model used to represent a set of concepts within a domain and the relationships between them. They usually appear in the form of taxonomies and ontologies. For example, Taibi et al. (2017) used the DBpedia knowledge base for cross-language interlinking based on the concepts recognized in the user's query.

Resource Cognitive Level (RCL) represents the cognitive level required to under- 
stand a resource to be returned by the search. It may be a representation of difficulty calculated from feedback or adjusted by experts in the knowledge domain. However, it is usually associated with levels of a taxonomy. For example, Al-Tawil et al. (2019) used the cognitive level from Revised Bloom's Taxonomy (Krathwohl, 2002) to assess learning experiences over information seeking and search tasks.

Resource Features $(R F)$ are variables related to characteristics of the resources returned by a search system. For example, Syed and Collins-Thompson (2018) considered web document features such as vocabulary difficulty, word count, number of images, paragraph density, among others, in order to identify the relationship of these variables with the final learning result in the short or long term. Shi et al. (2019) used this type of variable to investigate the correlation between them and the quality of educational videos. Document features could influence on learning experience and in some other factors such as motivation (Rieh et al., 2016) and long term memory stimulus (Smith and Rieh, 2019).

We propose to organize these variables into three bigger dimensions, namely, User Dimension, Interactive Dimension and Knowledge Domain Dimension:

User Dimension: This dimension encompasses the variables related to the searcher who intends to learn. Variables related to this dimension are commonly used to promote contextualized recommendations during information retrieval or to understand user's search behavior. We allocated User Prior Knowledge and Demographic Information here;

Interaction Dimension (ID): This dimension encompasses the variables that represent the interaction between the searcher and information enabled by a search tool during the execution of learning-driven tasks. We allocated Exploratory Search Activities, Activities Variables and Search Engine Feedback here;

Knowledge Domain Dimension (KDD): This dimension is related to the knowledge domain base where the user is searching to acquire learning. The search engine implicitly provides these variables, without the user's concern, or explicitly when the user is aware of the system's action. We allocated Knowledge Domain Representation, Resource Cognitive Level, and Resource Features here.

\section{Concluding Remarks}

This paper presented a systematic mapping of the literature on SAL proposals based on the variables that were observed to be used as input to support searching as a learning process. Based on these variables, we inferred three dimensions to classify the studies, namely, User Dimension, Interaction Dimension and Knowledge Domain Dimension.

The classification of the studies (Table 1), provides some insights about SAL. We can note the many of the works consider the users' personal information for a possible personalization of the information. It is in line with other areas, focused on online learning, that postulate that instruction should be individualized according to the characteristics and needs of each student. Also, the use of prior knowledge indicates that it is necessary to understand the user's current state of knowledge to offer more assertive resources since the complexity of the information directly influences learning and motivation. However, informal systems need to create strategies to gather and use such information to provide appropriate instruction if a learning support system during the search process is the target. 
IX Congresso Brasileiro de Informática na Educação (CBIE 2020)

Anais do XXXI Simpósio Brasileiro de Informática na Educação (SBIE 2020)

\begin{tabular}{|c|c|c|}
\hline Dimension & Variables & Papers \\
\hline \multirow[t]{2}{*}{$\begin{array}{l}\text { User } \\
\text { Dimension }\end{array}$} & PK & $\begin{array}{l}\text { (Lu and Hsiao, 2017), (Taibi et al., 2017), (Syed and Collins-Thompson, 2018), (Syed } \\
\text { and Collins-Thompson, 2016), (Jansen et al., 2009), (Yu et al., 2018), (Al-Tawil et al., } \\
\text { 2019), (Rieh et al., 2012), (Sendurur et al., 2019) (Tibau et al., 2018), (Azpiazu et al., } \\
\text { 2017), (Karanam and van Oostendorp, 2016), (Wilson et al., 2016), (Crescenzi, 2016), } \\
\text { (Mao et al., 2016), (Bhattacharya and Gwizdka, 2019), (Ibieta et al., 2019), (Biletskiy } \\
\text { et al., 2009), (Pereira et al., 2019) }\end{array}$ \\
\hline & DI & $\begin{array}{l}\text { (Taibi et al., 2017), (Azpiazu et al., 2017), (Ibieta et al., 2019), (Yilmaz et al., 2019), } \\
\text { (Biletskiy et al., 2009), (Lu and Hsiao, 2017), (Moraes et al., 2018) }\end{array}$ \\
\hline \multirow{3}{*}{$\begin{array}{l}\text { Interaction } \\
\text { Dimension }\end{array}$} & ESA & $\begin{array}{l}\text { (Tibau et al., 2018), (Lu and Hsiao, 2017), (Moraes et al., 2018), (Ghosh et al., 2018), } \\
\text { (Bhattacharya and Gwizdka, 2019), (Yu et al., 2018), (Ibieta et al., 2019), (Vakkari et al., } \\
\text { 2019), (Biletskiy et al., 2009) }\end{array}$ \\
\hline & AV & $\begin{array}{l}\text { (Bhattacharya and Gwizdka, 2019), (Yu et al., 2018), (Rieh et al., 2012), (Karanam and } \\
\text { van Oostendorp, 2016), (Ibieta et al., 2019), (Vakkari et al., 2019), (Wilson and Wilson, } \\
\text { 2013), (Maxwell et al., 2019) }\end{array}$ \\
\hline & SEF & $\begin{array}{l}\text { (Azpiazu et al., 2017), (Syed and Collins-Thompson, 2016), (Weingart and Eickhoff, } \\
\text { 2016), (Ibieta et al., 2019) }\end{array}$ \\
\hline \multirow{3}{*}{$\begin{array}{l}\text { Knowledge } \\
\text { Domain } \\
\text { Dimension }\end{array}$} & KDR & $\begin{array}{l}\text { (Taibi et al., 2017), (Al-Tawil et al., 2019), (Biletskiy et al., 2009), (Syed and Collins- } \\
\text { Thompson, 2018), (Ibieta et al., 2019), (Ghosh et al., 2018), (Karanam and van Oosten- } \\
\text { dorp, 2016), (Vakkari et al., 2019), (Tibau et al., 2019a), (Tolmachova et al., 2019) }\end{array}$ \\
\hline & $\mathrm{RCL}$ & $\begin{array}{l}\text { (Ghosh et al., 2018), (Syed and Collins-Thompson, 2018), (Syed and Collins-Thompson, } \\
\text { 2016), (Smith and Rieh, 2019), (Yu et al., 2018), (Al-Tawil et al., 2019), (Pereira et al., } \\
\text { 2019) }\end{array}$ \\
\hline & $\mathrm{RF}$ & $\begin{array}{l}\text { (Syed and Collins-Thompson, 2018), (Biletskiy et al., 2009), (Moraes et al., 2018), (Taibi } \\
\text { et al., 2017), (Ghosh et al., 2018), (Weingart and Eickhoff, 2016), (Vakkari et al., 2019), } \\
\text { (Wilson and Wilson, 2013), (Shi et al., 2019), (Fails et al., 2019) }\end{array}$ \\
\hline
\end{tabular}

The Interaction Dimension introduces a new layer of observation when comparing SAL to other areas that target only the learner and the content. This dimension show us that users must be assisted during the entire search session as learning takes place during this exploratory interaction. Future research should aim at this dimension in order to understand how search engines can support users in choosing better search terms, to avoid irrelevant content according to their intents, and to be aware when they are having comprehension problems or is struggling to formulate new queries and then act accordingly. Finally, considering the Knowledge Domain Dimension, we believe that semantic representations should be the basis of representation to improve the assimilation of user questions and to deliver richer content. Besides, in agreement with Hoppe et al. (2018), we believe that multimodal characteristics of multimedia content should be explored since this type of content is widely accepted in the learning context, especially in higher levels of the Bloom's Taxonomy (i.e., analyze, evaluate and create) (Krathwohl, 2002).

As future work, we intend to verify the influence of these variables on the learning evolution of users during search activities. Furthermore, it is important to correlate these variables with learning paradigms since Searching as Learning is about learning, so the proposed solutions must be based on learning theories and approaches.

\section{Acknowledgment}

This study was financed in part by the 'National Council for Scientific and Technological Development (CNPq) - Brazil' - Process 315374/2018-7, Project 'Searching as Learning: the information search as a tool for learning'. And was financed in part by the Coordination of Superior Level Staff Improvement - Brazil (CAPES) - Finance Code 001. 
IX Congresso Brasileiro de Informática na Educação (CBIE 2020)

Anais do XXXI Simpósio Brasileiro de Informática na Educação (SBIE 2020)

\section{References}

Al-Tawil, M., Dimitrova, V., and Thakker, D. (2019). Using knowledge anchors to facilitate user exploration of data graphs. Semantic Web, pages 1-30.

Azpiazu, I. M., Dragovic, N., Pera, M. S., and Fails, J. A. (2017). Online searching and learning: Yum and other search tools for children and teachers. Information Retrieval Journal, 20:524-545.

Bates, M. J. et al. (1989). The design of browsing and berrypicking techniques for the online search interface. Online review, 13(5):407-424.

Bhattacharya, N. and Gwizdka, J. (2019). Measuring learning during search: Differences in interactions, eye-gaze, and semantic similarity to expert knowledge. In Proceedings of the 2019 Conference on Human Information Interaction and Retrieval, pages 63-71. ACM.

Biletskiy, Y., Baghi, H., Keleberda, I., and Fleming, M. (2009). An adjustable personalization of search and delivery of learning objects to learners. Expert Systems with Applications, 36(5):9113-9120.

Crescenzi, A. (2016). Metacognitive knowledge and metacognitive regulation in timeconstrained in information search. In SAL@SIGIR, volume 1647, page 0.

Fails, J. A., Pera, M. S., Anuyah, O., Kennington, C., Wright, K. L., and Bigirimana, W. (2019). Query formulation assistance for kids: What is available, when to help \& what kids want. In Proceedings of the 18th ACM International Conference on Interaction Design and Children, pages 109-120.

Ghosh, S., Rath, M., and Shah, C. (2018). Searching as learning: Exploring search behavior and learning outcomes in learning-related tasks. In Proceedings of the 2018 Conference on Human Information Interaction \& Retrieval, pages 22-31.

Gossen, T., Hempel, J., and Nürnberger, A. (2013). Find it if you can: usability case study of search engines for young users. Personal and Ubiquitous Computing, 17(8):15931603.

Gwizdka, J., Hansen, P., Hauff, C., He, J., and Kando, N. (2016). Search as learning (sal) workshop 2016. In Proceedings of the 39th International ACM SIGIR conference on Research and Development in Information Retrieval, pages 1249-1250. ACM.

Hoppe, A., Holtz, P., Kammerer, Y., Yu, R., Dietze, S., and Ewerth, R. (2018). Current challenges for studying search as learning processes. In 7th Workshop on Learning \& Education with Web Data (LILE2018), in conjunction with ACM Web Science 2018, Amsterdam, NL, 27 May, 2018.

Ibieta, A., Hinostroza, J. E., and Labbé, C. (2019). Improving students' information problem-solving skills on the web through explicit instruction and the use of customized search software. Journal of Research on Technology in Education, 0:1-22.

Inhelder, B. and Piaget, J. (1958). The Growth of Logical Thinking from Childhood to Adolescence: An Essay on the Construction of Formal Operational Structures. Developmental psychology]. Routledge.

Jansen, B. J., Booth, D., and Smith, B. (2009). Using the taxonomy of cognitive learning to model online searching. Information Processing \& Management, 45(6):643-663.

Karanam, S. and van Oostendorp, H. (2016). Integrating domain knowledge differences into modeling user clicks on search result pages. In SAL@SIGIR.

Kitchenham, B. and Charters, S. (2007). Guidelines for performing systematic literature reviews in software engineering. Information and software technology. 
IX Congresso Brasileiro de Informática na Educação (CBIE 2020)

Anais do XXXI Simpósio Brasileiro de Informática na Educação (SBIE 2020)

Krathwohl, D. R. (2002). A revision of bloom's taxonomy: An overview. Theory into practice, 41(4):212-218.

Lu, Y. and Hsiao, I.-H. (2017). Personalized information seeking assistant (pisa): from programming information seeking to learning. Information Retrieval Journal, 20(5):433-455.

Lucchese, C., Nardini, F. M., Perego, R., Trani, R., and Venturini, R. (2018). Efficient and effective query expansion for web search. In Proceedings of the 27th ACM International Conference on Information and Knowledge Management, pages 1551-1554.

Machado, M., Pinelli, C., and Siqueira, S. (2019). A evolução da área de busca como um processo de aprendizagem com base em um mapeamento sistemático. In Anais dos Workshops do Congresso Brasileiro de Informática na Educação, page 833.

Machado, M. d. O. C., Bravo, N. F. S., Martins, A. F., Bernardino, H. S., Barrere, E., and de Souza, J. F. (2020). Metaheuristic-based adaptive curriculum sequencing approaches: a systematic review and mapping of the literature. Artificial Intelligence Review, pages 1-44.

Mao, J., Liu, Y., Zhang, M., and Ma, S. (2016). How does domain expertise affect users' search processes in exploratory searches? In SAL@ SIGIR.

Marchionini, G. (2006). Exploratory search: from finding to understanding. Communications of the ACM, 49(4):41-46.

Maxwell, D., Azzopardi, L., and Moshfeghi, Y. (2019). The impact of result diversification on search behaviour and performance. Information Retrieval Journal, 22(5):422446.

Moraes, F., Putra, S. R., and Hauff, C. (2018). Contrasting search as a learning activity with instructor-designed learning. In Proceedings of the 27th ACM International Conference on Information and Knowledge Management, pages 167-176. ACM.

Moreno-Marcos, P. M., Pong, T.-C., Muñoz-Merino, P. J., and Kloos, C. D. (2020). Analysis of the factors influencing learners' performance prediction with learning analytics. IEEE Access, 8:5264-5282.

Pereira, C. K., Medeiros, J. F., Siqueira, S. W., and Nunes, B. P. (2019). How complex is the complexity of a concept in exploratory search. In 2019 IEEE 19th International Conference on Advanced Learning Technologies (ICALT), volume 2161, pages 17-21. IEEE.

Pirolli, P. and Card, S. (1999). Information foraging. Psychological Review, 106:643-675.

Rieh, S. Y., Collins-Thompson, K., Hansen, P., and Lee, H.-J. (2016). Towards searching as a learning process: A review of current perspectives and future directions. Journal of Information Science, 42(1):19-34.

Rieh, S. Y., Kim, Y.-M., and Markey, K. (2012). Amount of invested mental effort (aime) in online searching. Information Processing \& Management, 48(6):1136-1150.

Sendurur, E., Efendioğlu, E., Senturk, H., and Calıskan, N. (2019). High achievers' web searching behaviors and patterns in two different task types. Journal of Educational Multimedia and Hypermedia, 28(2):217-238.

Shi, J., Otto, C., Hoppe, A., Holtz, P., and Ewerth, R. (2019). Investigating correlations of automatically extracted multimodal features and lecture video quality. In Proceedings of the 1st International Workshop on Search as Learning with Multimedia Information, pages 11-19.

Smith, C. L. and Rieh, S. Y. (2019). Knowledge-context in search systems: toward information-literate actions. In Proceedings of the 2019 Conference on Human Information Interaction and Retrieval, pages 55-62. 
IX Congresso Brasileiro de Informática na Educação (CBIE 2020)

Anais do XXXI Simpósio Brasileiro de Informática na Educação (SBIE 2020)

Soulier, L. and Tamine, L. (2017). On the collaboration support in information retrieval. ACM Computing Surveys, 50(4):1-34.

Syed, R. and Collins-Thompson, K. (2016). Optimizing search results for educational goals: Incorporating keyword density as a retrieval objective. In SAL@SIGIR.

Syed, R. and Collins-Thompson, K. (2017). Retrieval algorithms optimized for human learning. In Proceedings of the 40th International ACM SIGIR Conference on Research and Development in Information Retrieval, pages 555-564.

Syed, R. and Collins-Thompson, K. (2018). Exploring document retrieval features associated with improved short-and long-term vocabulary learning outcomes. In Proceedings of the 2018 Conference on Human Information Interaction \& Retrieval, pages 191-200.

Taibi, D., Fulantelli, G., Marenzi, I., Nejdl, W., Rogers, R., and Ijaz, A. (2017). Sarweb: a semantic web tool to support search as learning practices and cross-language results on the web. In 2017 IEEE 17th International Conference on Advanced Learning Technologies (ICALT), pages 522-524. IEEE.

Tibau, M., Siqueira, S., and Nunes, B. P. (2019a). A comparison between entity-centric knowledge base and knowledge graph to represent semantic relationships for searching as learning situations. In Anais dos Workshops do Congresso Brasileiro de Informática na Educação, volume 8, page 823.

Tibau, M., Siqueira, S. W., Nunes, B. P., Bortoluzzi, M., Marenzi, I., and Kemkes, P. (2018). Investigating users' decision-making process while searching online and their shortcuts towards understanding. In International Conference on Web-Based Learning, pages 54-64. Springer.

Tibau, M., Siqueira, S. W., Nunes, B. P., Nurmikko-Fuller, T., and Manrique, R. F. (2019b). Using query reformulation to compare learning behaviors in web search engines. In 2019 IEEE 19th International Conference on Advanced Learning Technologies, volume 2161, pages 219-223. IEEE.

Tolmachova, T., Xu, L., Marenzi, I., and Gadiraju, U. (2019). Visualizing search history in web learning. In International Conference on Web-Based Learning, pages 229-240. Springer.

Vakkari, P. (2016). Searching as learning: A systematization based on literature. Journal of Information Science, 42(1):7-18.

Vakkari, P., Völske, M., Potthast, M., Hagen, M., and Stein, B. (2019). Modeling the usefulness of search results as measured by information use. Information Processing \& Management, 56:879-894.

Weingart, N. and Eickhoff, C. (2016). Retrieval techniques for contextual learning. In SAL@SIGIR.

Wilson, M. J. and Wilson, M. L. (2013). A comparison of techniques for measuring sensemaking and learning within participant-generated summaries. Journal of the American Society for Information Science and Technology, 64:291-306.

Wilson, M. L., Ye, C., Twidale, M. B., Grasse, H., Rosenthal, J., and McKittrick, M. (2016). Search literacy: Learning to search to learn. In SAL@SIGIR, volume 1647.

Yilmaz, T., Ozcan, R., Altingovde, I. S., and Ulusoy, H. (2019). Improving educational web search for question-like queries through subject classification. Information Processing \& Management, 56:228-246.

Yu, R., Gadiraju, U., Holtz, P., Rokicki, M., Kemkes, P., and Dietze, S. (2018). Predicting user knowledge gain in informational search sessions. In The 41st International ACM SIGIR Conference on Research \& Development in Information Retrieval, pages 75-84. 